\title{
Improved Specimen Preparation and SEM Imaging Reveal the Morphology of a West African Sorghum Resistant to Storage Insects.
}

\author{
M.W. Pendleton ${ }^{1}$, E.A. Ellis ${ }^{1}$, B.B. Pendleton ${ }^{2}$, and N.Y. Diarisso ${ }^{3}$ \\ 1. Microscopy \& Imaging Center, Texas A\&M University, Mail Stop 2257, College Station, TX 77843- \\ 2257, U.S.A. \\ 2. Agricultural Sciences, Box 60998, West Texas A\&M University, Canyon, TX 79016-0001, U.S.A. \\ 3. Institut D'Economie Rurale, B.P. 258, Bamako, Mali
}

The maize weevil, Sitophilus zeamais Motschulsky, is the most damaging pest worldwide of stored grain. Chitio [1] determined resistance to maize weevils of 7 of the genotypes of sorghum, Sorghum bicolor (L.) Moench, discussed in this work. Diarisso determined resistance of Seguifa sorghum in Mali. Shallow cuts ( $1 \mathrm{~mm}$ depth) were made with a razor on kernels of 7 genotypes of sorghum (except Seguifa), fixed in $2.5 \%$ glutaraldehyde - 1\% acrolein in HEPES buffer ( $\mathrm{pH} 7.3$ ), followed by $1 \%$ osmium in HEPES buffer ( $\mathrm{pH} 7.3$ ). Methanol dehydration was done at 5\% steps, followed by 3 changes of Hexamethyl-disilazane (HMDS). Dried kernels were polymerized in low-viscosity resin (6 changes), cut by diamond knife in cross section with a Porter Blum MT-1, and coated with iodine (IKI) vapor [2]. Block faces were coated with $35-50 \mathrm{~nm}$ of carbon by Cressington 308R evaporative coater. Kernels were imaged in both secondary and backscatter modes in a JEOL JSM-6400 SEM (15 kV and $15 \mathrm{~mm}$ working distance). Seguifa kernels were initially razor cut in cross-section, then fixed, dehydrated, and polymerized in the same way. Sectioning was done by a diamond knife with a Porter-Blum MT-1 just below the initial razor cut and the sectioned blocks were coated with iodine (IKI) vapor [2] and also carbon coated $(50 \mathrm{~nm})$ using a Cressington 308R coater. This procedure allowed backscatter (Fig. 1A) and secondary SEM images (Fig. 1B) of the Seguifa kernel to be made. A similar block face SEM method was demonstrated by Deerinck et al. [3]. The backscatter (Fig. 1A) SEM image of the Seguifa kernel demonstrates that while the resin held loose material in place within the block face, the surface of the resin is not visible as it is in the secondary image (Fig. 1B). The backscatter SEM image (Fig. 2A) of the Segaolane sorghum kernel shows voids caused by the absence of resin on the block face during sectioning. Backscatter SEM images of Seguifa kernels and the other 7 sorghum genotypes (including Segaolane) [4] demonstrated vivid compositional contrast (bright areas) in starch to allow accurate measurements to the seed coat, although this contrast was muted in secondary SEM mode (Fig. 1B).

A correlation was demonstrated for the genotypes of sorghum so that as the depth of the starch areas (with bound iodine (IKI) as a marker) measured to the seed coat increased, the percentage of weight loss and damage scores of stored grain infested with maize weevils decreased (Fig. 2B).

\section{References}

[1] F Chitio, M.S. Thesis, West Texas A\&M University, (2004).

[2] E Ellis and M Pendleton, Microscopy Today 15 (2007), p. 44.

[3] T Deerinck et al., Microscopy and Microanalysis 16 (suppl. 2) (2010), p. 1138.

[4] M Pendleton et al., Microscopy and Microanalysis 17 (suppl. 2) (2011), p. 250.

[5] Research was supported in part by the Sorghum, Millet \& Other Grains Collaborative Research Support Program (INTSORMIL CRSP), USAID Grant No. LAG-G-00-96-900009-00. 

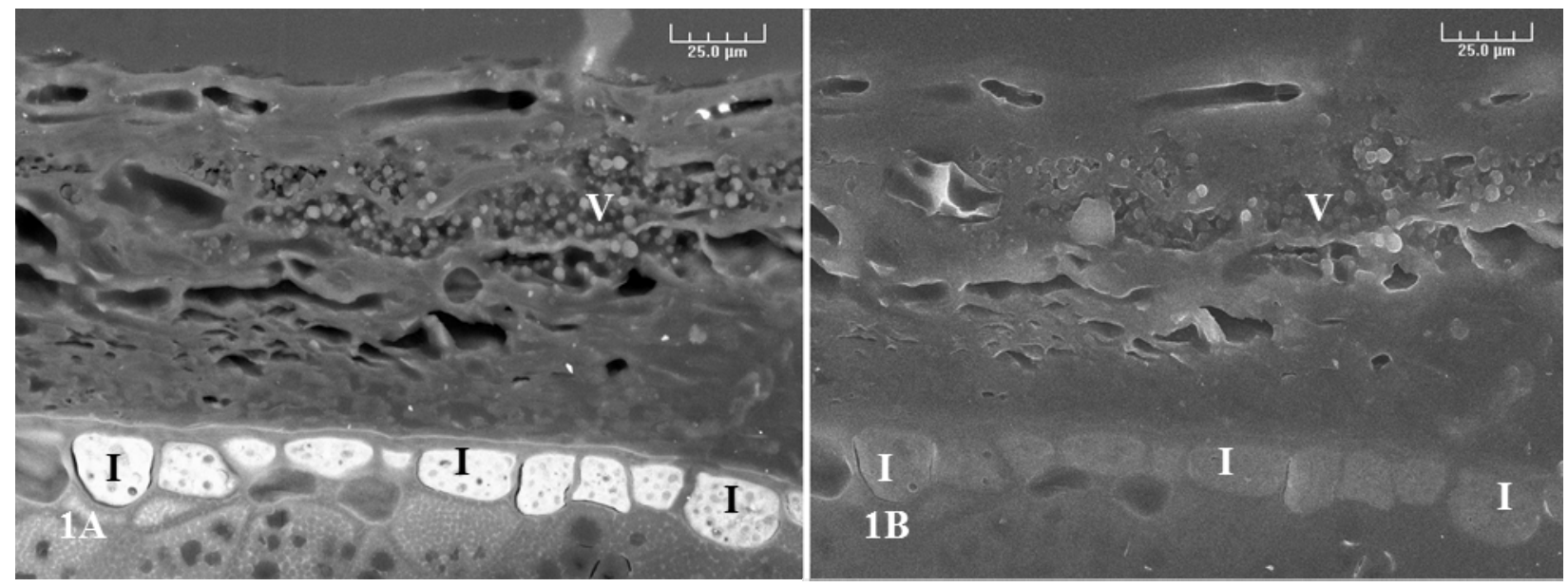

Figure 1A. Seguifa backscatter SEM image. Figure 1B. Secondary SEM image of same block face as in Fig. 1A. Resin on block faces IKI and carbon coated. Iodine (I) shown as very brilliant white areas in Fig. 1A (backscatter mode) due to compositional contrast while these same areas (I) are only slightly lighter in Fig. 1B (secondary mode). Backscatter SEM signals pass through resin so the details in voids (V) are visible (Fig. 1A) but are not readily seen (V) in secondary mode (Fig. 1B). Scale bars $=25 \mu \mathrm{m}$.
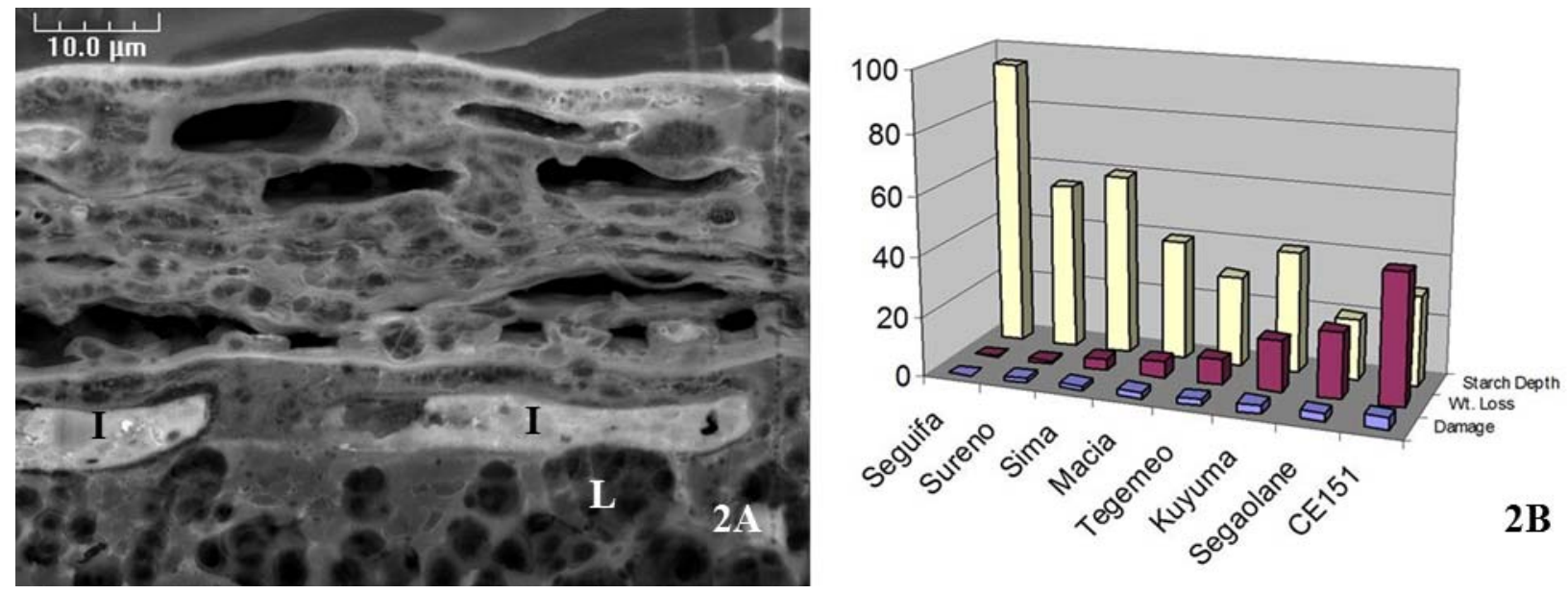

Figure 2A. Segaolane backscatter SEM image of kernel razor cut in cross-section (no resin on block face) producing loss of material (L) at the surface. Iodine (I) is shown as very bright areas in Fig. 2A because of compositional contrast in backscatter mode. Confirmation of iodine concentration in the bright areas (Figs. 1A and 2A) demonstrated by PGT (Bruker) SEM-EDS analysis. Scale bar $=10 \mu \mathrm{m}$. Figure 2B. Graph demonstrates that greater depth to starch from the seed coat in sorghum kernels is generally related to greater resistance to damage by maize weevils in storage. Damage determined on scale of 1 to 5 , with 5 being the most damaged. Measurement of weight loss was in percentage and starch depth was measured in $\mu \mathrm{m}$ from seed coat. 